\title{
Ward Round - Recurrent anemia and infection in an HIV-positive woman
}

\section{Mulinda Nyirenda', Tom Latham², Simon Glover ${ }^{3}$}

1. Queen Elizabeth Central Hospital, Ministry of Health, Blantyre

2. Department of Medicine, College of Medicine, University of Malawi.

3. Department of Anatomy, College of Medicine, University of Malawi.

Corresponding author: Dr. T Latham, Dept of Medicine, College of Medicine, P/Bag 360, Blantyre, Malawi Email: tlatham@medcol.mw

A 22 year old woman presented to Queen Elizabeth Central Hospital with a three days history of fever, headache and vomiting, with progressive difficulty in swallowing.

\section{Past history}

Two weeks previously, she was admitted to hospital for severe anaemia $([\mathrm{Hb}] 3.2 \mathrm{~g} / \mathrm{dl})$ and probable sepsis, for which she was transfused three units of blood and received ceftriaxone and ciprofloxacin therapy. Her HIV test was found to be positive on that occasion, and she was commenced on cotrimoxazole prophylaxis. She was advised to start ART as soon as possible at her nearest health centre. She had not lost consciousness and had not had any convulsions.

\section{On examination for the present re-admission:}

The patient was weak, lethargic and very pale. The temperature was $38.60 C$, blood pressure $80 / 40$, pulse rate $138 / \mathrm{min}$ and breathing rate $42 / \mathrm{min}$. Severe oral thrush was noted. Lymph nodes were not enlarged. Chest and heart examinations were normal. There was generalized abdominal tenderness and the spleen was $10 \mathrm{~cm}$ palpable. The skin and extremities were normal. There was moderate neck stiffness. No other neurological abnormality was found.

Ophthalmoscopy revealed severe retinopathy (see Figs 1-3)

\section{Investigations}

Full blood count: [Hb] $1.9 \mathrm{~g} / \mathrm{dl}$, wbc $4.2 \times 10^{9} / 1$, (neutrophils $39 \%$, lymph $39 \%$ ), platelets $21 \times 10^{9} / 1$. Thin film: red cells normocytic; no malaria parasites seen; for additional cells see Fig 4.

CD4 count 377/ul. Random blood glucose $60 \mathrm{mg} / \mathrm{dl}$. Lumbar puncture: opening pressure $6 \mathrm{~cm}$, fluid cloudy and slightly blood stained. Microscopy of CSF: 560 red blood cells/ul, glucose $48 \mathrm{mg} / \mathrm{dl}$, protein $97 \mathrm{mg} / \mathrm{dl}$; Indian ink t، negative for cryptococci. There was no growth on culti after 48 hours of incubation. Blood culture: no grow Ultrasonography of abdomen: mild hepatosplenomega normal kidneys, no lymphadenopathy

\section{Treatment and progress}

The patient was started on ceftriaxone $2 \mathrm{~g}$ BD IV, fluconazc $200 \mathrm{mg}$ od and paracetamol. Five units of blood we transfused. Cotrimoxazole prophylaxis was continued. ( the fourth day the patient had repeated convulsions, whi... were controlled by administration of phenobarbitone.

A repeat full blood count 10 days later showed improvement in white cell count to $6.8 \times 10^{9} / 1$ (neurophils $52 \%$ lymph $33 \%$ ), and in [Hb] to $5.2 \mathrm{~g} / \mathrm{dl}(\mathrm{MCV} 78, \mathrm{MCH} 26.9)$; but platelets remained low at $20 \times 10^{9} / 1$.

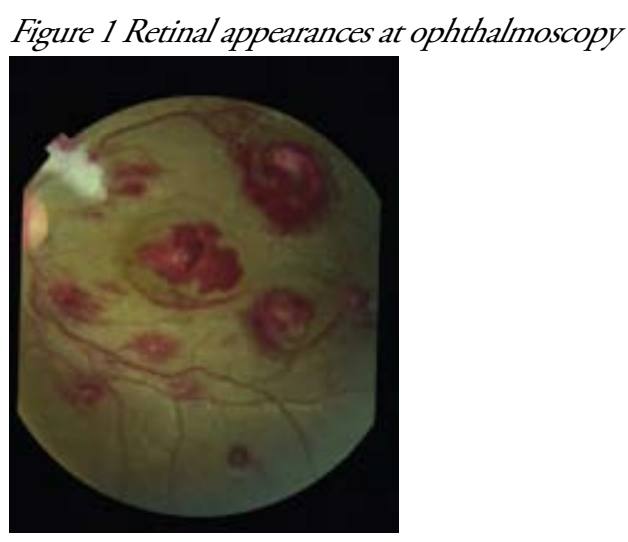

Figure 2 Retinal appearances at ophthalmoscopy

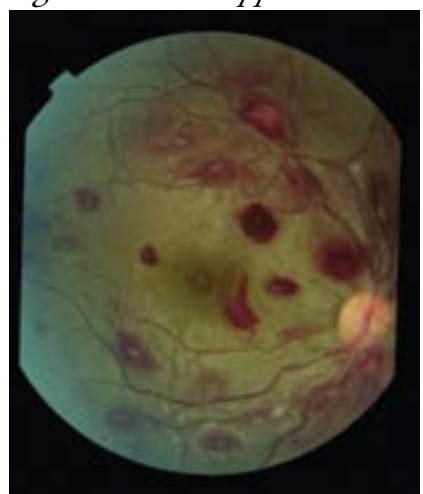

Figure 3 Retinal appearances at ophthalmoscopy

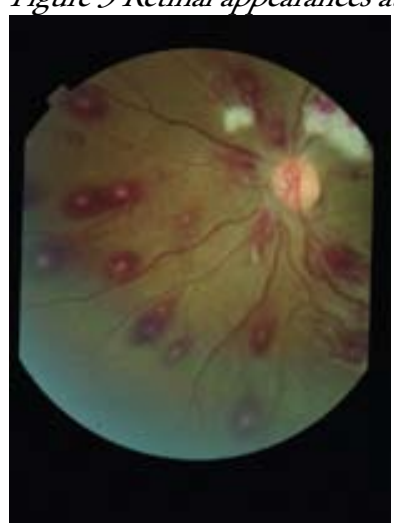

Figure 4

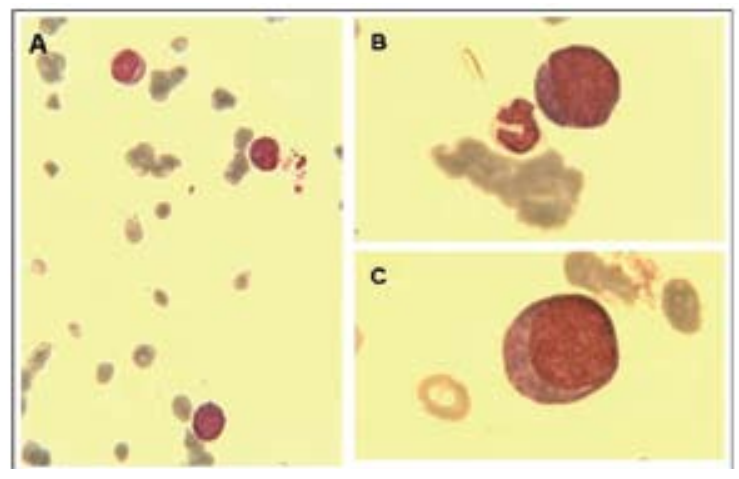

1. Describe the ophthalmoscopic findings (Figs 1-3).

2. What do the peripheral blood films show (Fig 4)?

3. What are the likely causes of her problem?

Turn to $\mathrm{pg} 88$ for a discussion of this case. 


\section{Ward Round-Recurrent anemia and infection in an HIV-positive woman}

\section{Continued from page 86}

The retinal appearances (Figs 1-3) include multiple whitecentred haemorrhages, two large white retinal lesions close to the left optic disk, and retinal haemorrhage and fluid just nasal to the $\mathrm{R}$ fovea. All of these changes are non-specific but are compatible with a severe systemic disease with thrombocytopenia as discussed below.

The peripheral blood film (Fig 4) shows the presence of blast cells with intense blue cytoplasm

\section{Discussion}

This patient presented with normocytic anaemia in the setting of pancytopenia, as noted on FBC and PBF. Pancytopenia in a patient with advanced stage HIV infection and sepsis is not uncommon and is often multifactorial especially in a tropical setting. Anaemia of inflammation, peripheral consumption due to sepsis itself, nutritional deficiencies, drugs, chronic infections such as TB, and the direct effect of HIV infection itself can all be factors and commonly coexist. It is entirely reasonable in the acute situation to assess response to treatment of sepsis and other identifiable conditions before seeking more specific bone marrow pathology.

However, the finding of blasts in the peripheral blood in the context of severe pancytopenia is almost diagnostic of acute leukaemia. Further characterization of an acute leukaemia as an acute lymphoid or myeloid leukaemia cannot be achieved based on light microscopy alone and requires immunophenotyping and/or molecular biology techniques. However, blasts with intense blue cytoplasm suggest a B cell origin. In the context of HIV infection, this is suggestive of Burkitt's lymphoma. Somewhat confusingly, Burkitt's lymphoma not infrequently presents with a purely leukaemic picture; indeed acute lymphoblastic leukaemia subtype L3 in the FAB classification is recognised to be biologically identical to Burkitt's lymphoma.

HIV infection is well known to predispose to development of haematological malignancies. The incidence of true acute leukaemias may be slightly increased in HIV infection ; a twofold higher incidence has been seen in AML in the developed worl ${ }^{d} 1$ and this is probably similar in Africa ${ }^{2}$. HIV infection increases the risk of development of a lymphoma 60 to 165 fold over that of the HIV seronegative population. Approximately 70 to 90 percent of the lymphomas encountered in HIV-infected patients are high grade lymphomas, particularly diffuse large B cell (DLBCL) and Burkitt's lymphomas (BL) ${ }^{3}$. Statistically therefore, an HIV positive adult presenting with a leukaemic blood picture is likely to have Burkitt's lymphoma. DLBCL usually arises in those with more advanced HIV infection, with a CD4 count typically $<100$ cells/microL, while BL may occur in the setting of a wide range of $\mathrm{CD} 4$ counts.

Burkitt's lymphoma has long been a disease of interest in sub-Saharan Africa. The classical form of the disease is prevalent in regions of Africa where malaria is endemic. This is a disease of children, classically affects the bones of the face or presents as abdominal or CNS tumours, and can account for over half of childhood malignancies in endemic regions ${ }^{4,5}$. It is an aggressive B cell malignancy, and is characterised by an ${ }^{8,14}$ chromosomal translocation creating a fusion gene between the cMYC oncogene and the IGH immunoglobulin heavy chain gene. The Epstein-Barr virus (EBV) is associated with almost all cases and is considered to play a causative role. In non-malarial areas, a form of the disease termed sporadic Burkitt's or Burkitt-like lymphoma occurs but is rare in non-immunosuppressed individuals. This has a different clinical course, rarely presenting with facial disease, but is still thought to be EBV associated. With the advent of HIV infection in Africa, Burkitt's lymphoma has become more prevalent in adults, but interestingly does not tend to follow the clinical course of the endemic form ${ }^{6}$.

Although Burkitt's-like lymphomas can respond rapidly to chemotherapy in the short term, survival is poor in both HIV positive and negative patients with conventional intensity regimens such as CHOP. There is commonly rapid relapse, in particular within the central nervous system. Two-year survivals of around $50-60 \%$ can be achieved with intensive chemotherapy regimens incorportating CNS treatment such as CODOX-M/IVAC, however such regimens cause prolonged neutropenia and need excellent supportive care to be able to achieve good outcomes ${ }^{7}$. With concomitant antiretroviral therapy, outcomes in HIV positive patients in the developed world are equivalent or slightly better than in HIV negative patients ${ }^{8}$. Interestingly, children with endemic Burkitt's can achieve long-term survival with single agent chemotherapy', suggesting that the biology of endemic Burkitt's may differ substantially from the sporadic disease.

\section{Extent of disease in our patient}

Our patient was noted to have systemic spread with bone marrow involvement. The absence of white blood cells in the CSF did not exclude leptomeningeal involvement. Occult CNS involvement is very frequently present in Burkitt's lymphoma, and concentration of cells in the CSF by cytospin is often required to detect this. It remains possible that the seizures in our patient were due to CNS disease. The raised CSF protein and low CSF glucose relative to serum levels is compatible with CNS lymphoma. The recurrent fever may be attributed to recurrent bacterial infections to which the patient was susceptible because of neutropenia. However, it is possible that the fever could be due to ' $\mathrm{B}$ ' symptoms of the malignancy.

\section{Treatment and prognosis}

The prognosis in this patient would have been poor with presentation of advanced disease (stage 4) even with optimal care facilities. In a resource poor setting, myelosuppression due to marrow infiltration is the limiting factor in considering chemotherapy. Even if her disease were responsive to the lower intensity chemotherapy regimens currently available, it would be necessary to provide supportive care for a prolonged period of neutropenia until recovery of normal haematopoiesis. Nevertheless, non-myelosuppressive agents 
such as vincristine and steroids (particularly dexamethasone in view of its CNS penetration) may play a useful role as an adjunct to symptomatic care; the disappearance of blasts from the peripheral blood after steroid therapy suggests her disease did show a response though this was short-lived.

The availability of community palliative care services enabled this patient to be discharged home with arrangements for ongoing symptomatic care. Despite the fact that we could not give curative treatment, it was beneficial in this patient to arrive promptly at a working pathological diagnosis, because this allowed us to make a positive decision to concentrate our efforts on palliative care.

\section{References}

1. Sutton, Laurent et al. (2001) Acute myeloid leukaemia in human immunodeficiency virus-infected adults: epidemiology, treatment feasibility and outcome. Haematological Malignancy British Journal of Haematology. 112(4-II):900-908

2. Mukiibi JM, Nyirenda CM, et al (2001) Leukaemia at Queen Elizabeth Central Hospital in Blantyre, Malawi E Afr Med J 78 (7): 349-354
3. http://www.uptodate.com.AIDS-relatedLymphomas:epidermiology and overview

4. Mutalima N, Molyneux E, et al.(2008) Associations between Burkitt Lymphoma among Children in Malawi and Infection with HIV, EBV and Malaria: Results from a Case-Control Study. PLoS ONE 3(6): e2505. doi:10.1371/journal.pone.0002505

5. Parkin MD, Sitas F et al (2008) Part I: Cancer in Indigenous Africans-burden, distribution, and trends. Lancet Oncol 9 683-692

6. http://www.uptodate.com. (2009) 'Clinical manifestations, pathologic features and diagnosis of Burkitt Lymphoma' and 'AIDS-related lymphomas:systemic lymphomas'

7. Oriol A, Ribera JM, et al (2005) Highly active antiretroviral therapy and outcome of AIDS-related Burkitt's lymphoma or leukemia. Results of the PETHEMA-LAL3/97 study. Haematologica 90 (7) 990-992

8. Hesseling PB, Molyneux EM, et al.(2008) Treating Burkitt's lymphoma in Malawi, Cameroon, and Ghana Lancet.oncology 2008 9; $512-513$

9. 9. Hesseling PB, Molyneux EM, et al.(2008) Treating Burkitt's lymphoma in Malawi, Cameroon, and Ghana Lancet.oncology 2008 9; $512-513$

See also: .http://www.clinicalcareoptions.com/Oncology/

AnnualUpdates/Lymphoma2005/Modules/lymphoma2005_aids/Pages/ Page1.aspx

\section{The inaugural Malawian science café}

On 8th April 2009, the first African science café was launched at College of Medicine Sports Complex Breezer Bar. This is an initiative of the Malawi Wellcome Liverpool Trust (MLW and the Malawi Medical Journal (MMJ). Khanyiwe Shawa, science communication officer (MLW), who was also the moderator of the event, introduced science cafés as live events where science, food and drink make excellent conversation. Science cafes are the brainchild of Duncan Dallas, UK with the African science cafes based on the European Cafe Scientifique model. They involve faceto-face interaction between the lay public and scientists. They offer an opportunity for scientists and the public to engage with each other to discuss current and or controversial health, science, research and technology topics in a relaxed environment. Scientist are encouraged to talk in plain language and the audience is advised to speak their mind, ask provoking questions, meet new friends, and talk with their mouths full.

The theme of the first science cafe was "Prevention of Mother to Child Transmission (PMTCT), how does it really happen?”. The two speakers Dr. Victor Mwapasa of the College of
Medicine and MLW and Dr.George Kafulafula of the College of Medicine and Johns Hopkins gave very lively presentations on how HIV is transmitted during pregnancy and how it can be prevented. They also presented current research findings

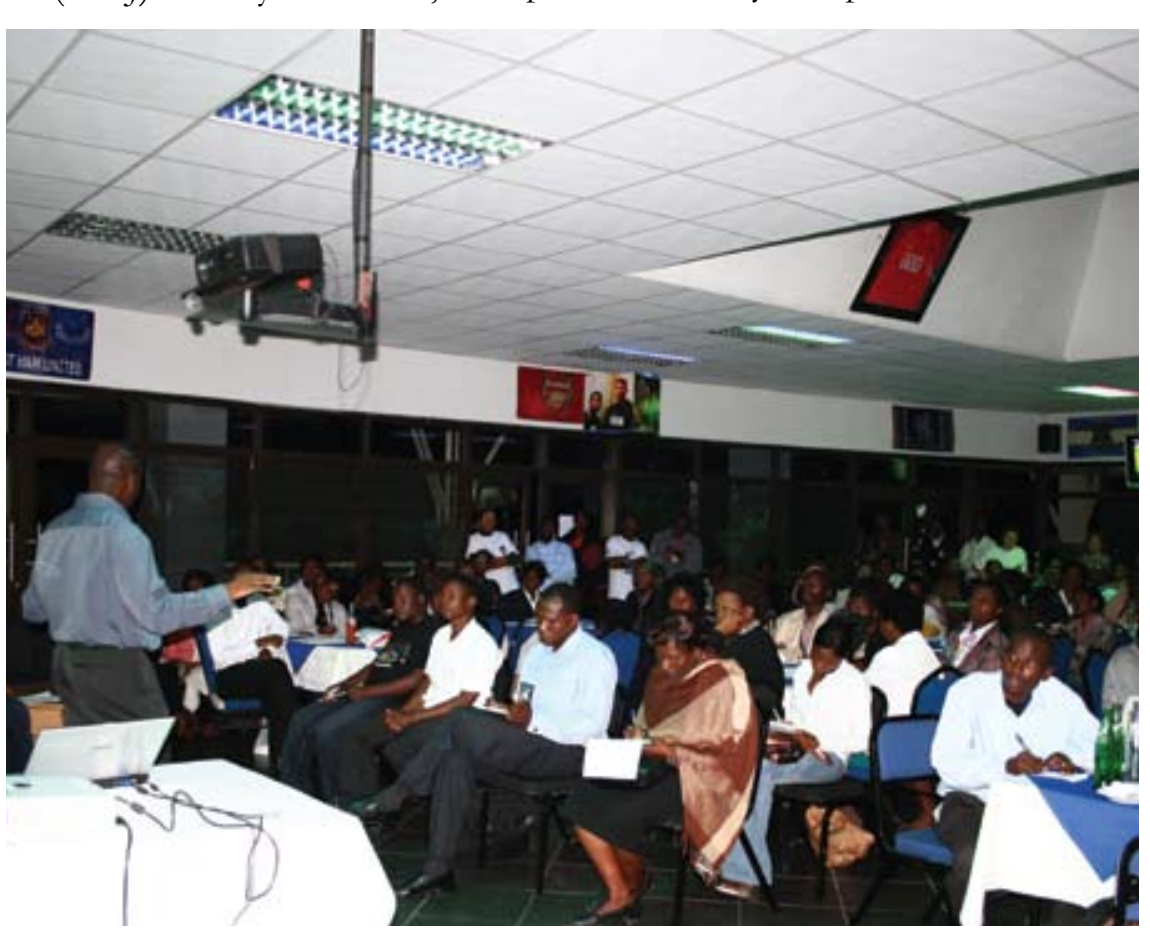
of PMTCT studies in Malawi.

The event was spiced up a with trivia quiz before the formal presentations, which saw members of the audience who got the questions right, winning MLW t-shirts. Drinks and snacks were also served to participants courtesy of the Centre for Reproductive Health, College of Medicine. Participants at the function were from all walks of life and included nurses, students, and engineers. The science cafe was well received by the public with calls for more science cafes on discordant couples, malaria and TB. 